AD-A087 248 AEROSPACE CORP EL SEEUNDO CA AEROPHYSICS LAB SENSITIVE MEASUREMENT OF PHOTON LIFETIME AND TRUE REFLECTANCES -ETC(U) JUL 80 J IM HERBELIN. J A MCKAY. IM A KWOK

$F / 620 / 6$ UNCLASSIFIED TR $=0000(5610)-2$ SD-TR $-80-48$ NL
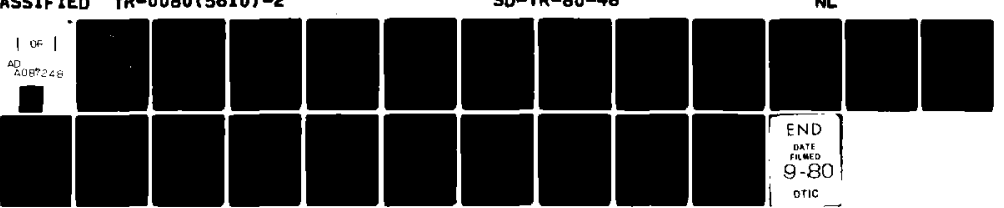


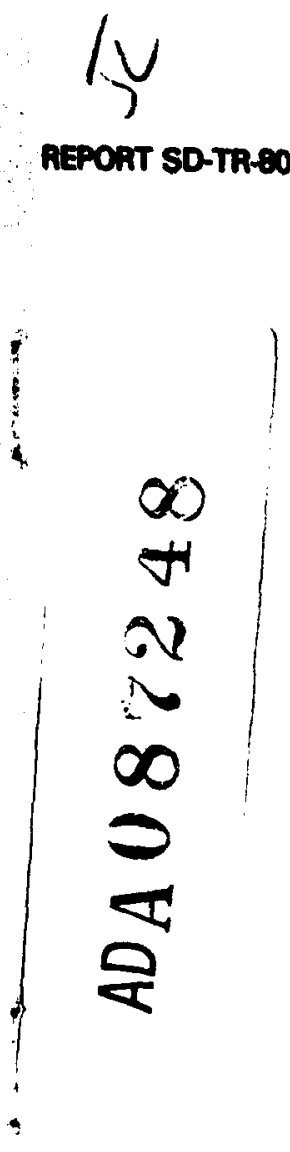

\section{Sensitive Measurement of Photon Lifetime and True Reflectances in an Optical Cavity by a Phase Shift Method}

J. M. HERBELIN, J. A. MCKAY, M. A. KWOK,

R. H. UEUNTEN, D. S. UREVIG, and D. J. SPENCER Aerophysics Laboratory Laboratory Operations

The Aerospace Corporation

El Segundo, Calif. 90245 and D. BENARD

Air Force Weapons Laboratory

Kirtland Air Force Base, N. Mex, 87117

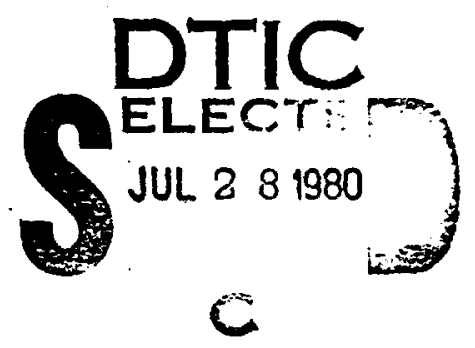

10 July 1980

Interim Report

APPROVED FOR PUBLIC RELEASE; DISTRIBUTION UNLIMITED
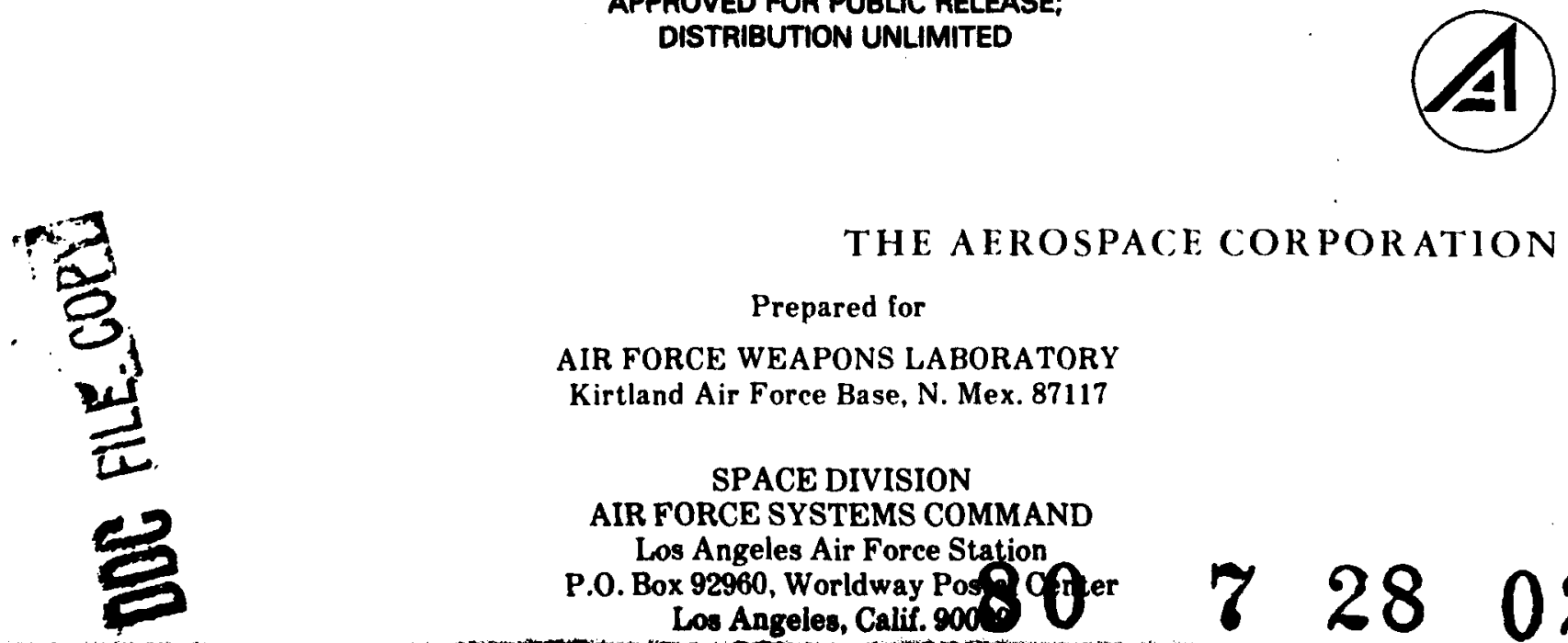

THE AEROSPACE CORPORATION

Prepared for

AIR FORCE WEAPONS LABORATORY

Kirtland Air Force Base, N. Mex. 87117

SPACE DIVISION

AIR FORCE SYSTEMS COMMAND

Los Angeles Air Force Station

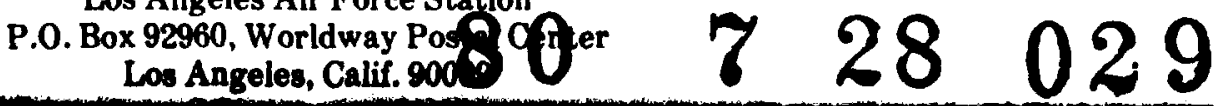


This interim report was submitted by The Aerospace Corporation, El Segundo, CA 90245, under Contract No. F04701-79-C-0080 with the Space Division, Deputy for Technology, P.O. Box 92960, Worldway Postal Center, Los Angeles, CA 90009. It was reviewed and approved for The Aerospace Corporation by W. R. Warren, Jr., Director, Aerophysics Laboratory. Lieutenant James C. Garcia, SD/Y LXT, was the project officer for Technology.

This report has been reviewed by the Public Affairs Office (PAS) and is releasable to the National Technical Information Service (N TIS). At NTIS, it will be available to the general public, including foreign nations.

This technical report has been reviewed and is approved for publicion. Publication of this report does not constitute Air Force approval of the report's findings or conclusions. It is published only for the exchange and stimulation of ideas.

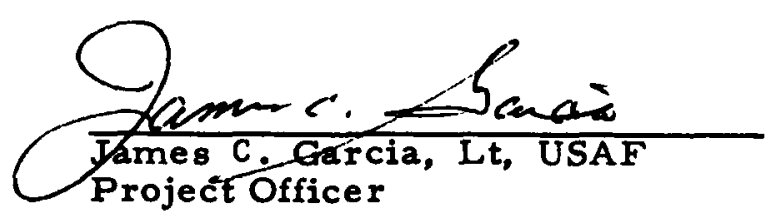

FOR THE COMMANDER

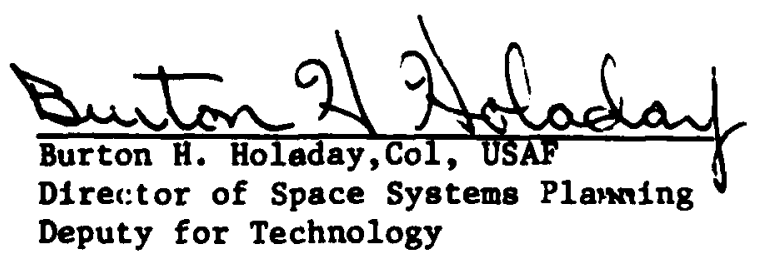

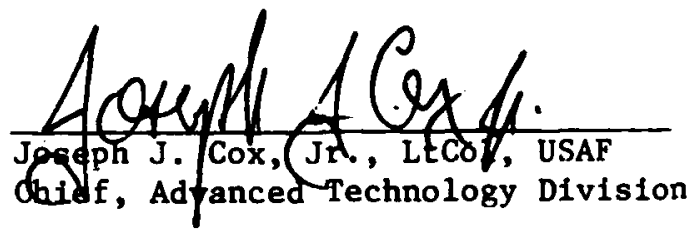

(1) 
UNCLASSIFIED
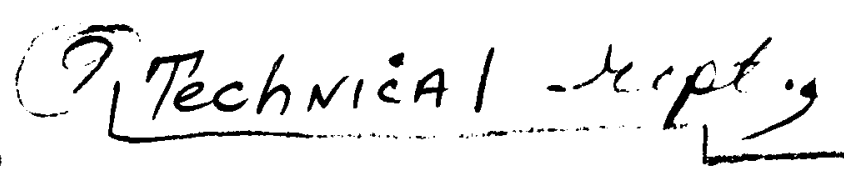

SECUAITY CLASSIFICATION OF THIS PAGE (WMan Date Entored)

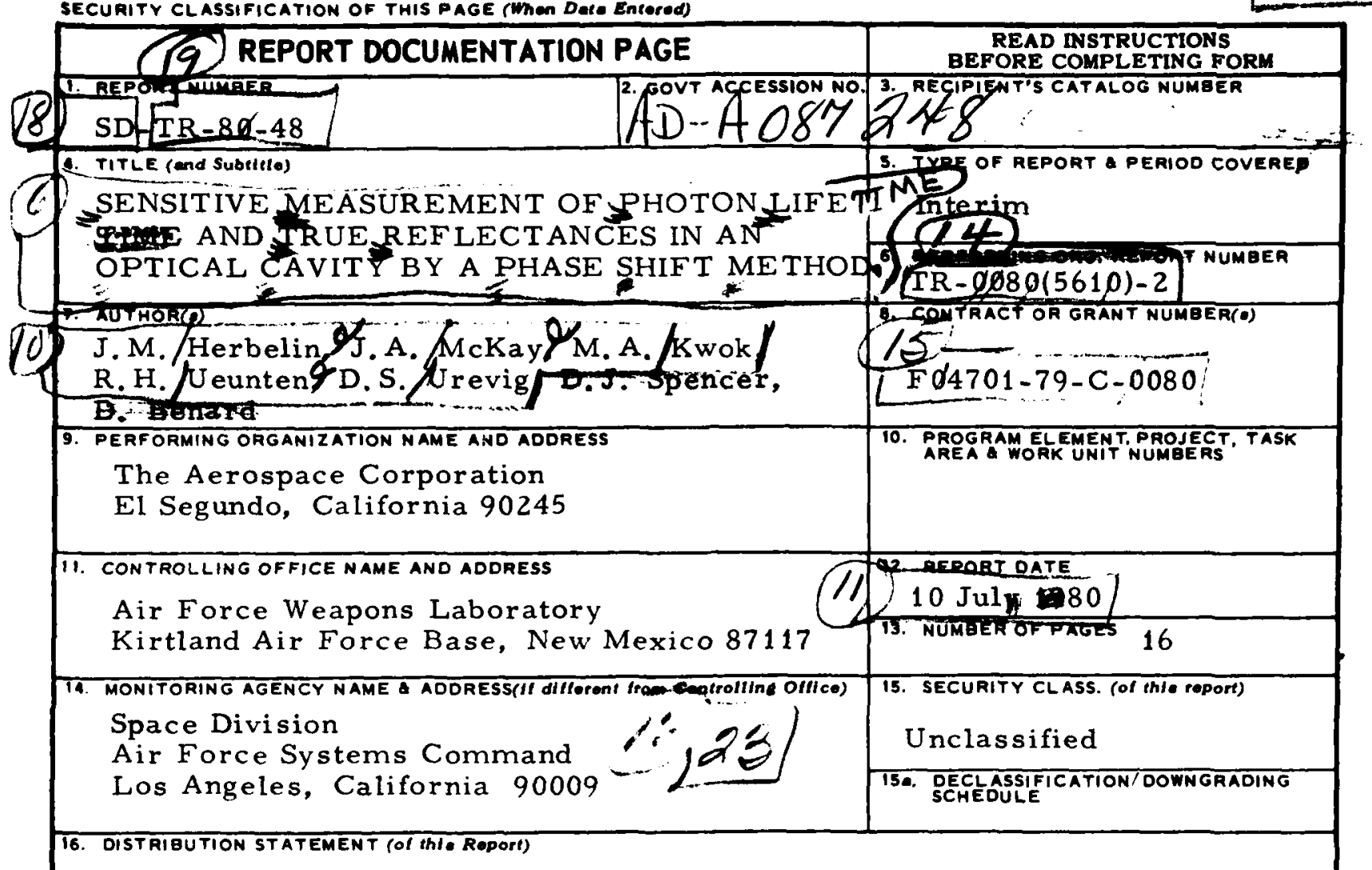

Approved for public release; distribution unlimited.

17. DISTRIBUTION STATEMENT (ol the ebetract entered in Block 20, If different from Roport)

18. SUPPLEMENTARY NOTES

19. KEY WOROS (Continue on reverce alde II neceesery end ldentlly by block number)

Optical resonator

Mirror reflectivity

Photon lifetime

Laser

20 ABSTAACT (Continue on reverse olde II neceecery and Identlly by block number)

A simplified method for measuring the effective photon lifetime in an optical resonator was developed. The technique requires the passage of a modulated continuous-wave laser beam through the resonator and the measurement of the resultant shift in the phase of the transmitted intensity. The method not only permits a quick and precise measurement of the mirror reflectances, but also permits these measurements to be in situ. Such an "on the spot" evaluation 
secunity CLASSIFICATION OF THIS PAOE(mmen Data Entered)

19. KEY WORDS (Continued)

20. AESTRACT (Continued)

It

Capability should be extremely useful in applications ranging from the investigation of new laser systems to the development of improved optical coatings. The method is also sensitive to the effects of absorption, scattering, and transmission from elements in the cavity. Cavity losses smaller than $100 \mathrm{ppm}$ were detected.

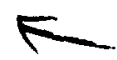


CONTENTS

I. INTRODUCTION . . . . . . . . . . . . . . 5

II. EXPERIMENT AND RESULTS. . . . . . . . . . . . . . 9

III. DISCUSSION . . . . . . . . . . . . . . 17

APPENDIX . . . . . . . . . . . . . . . . . . 19

REFERENCES. . . . . . . . . . . . . . . . . . . 23

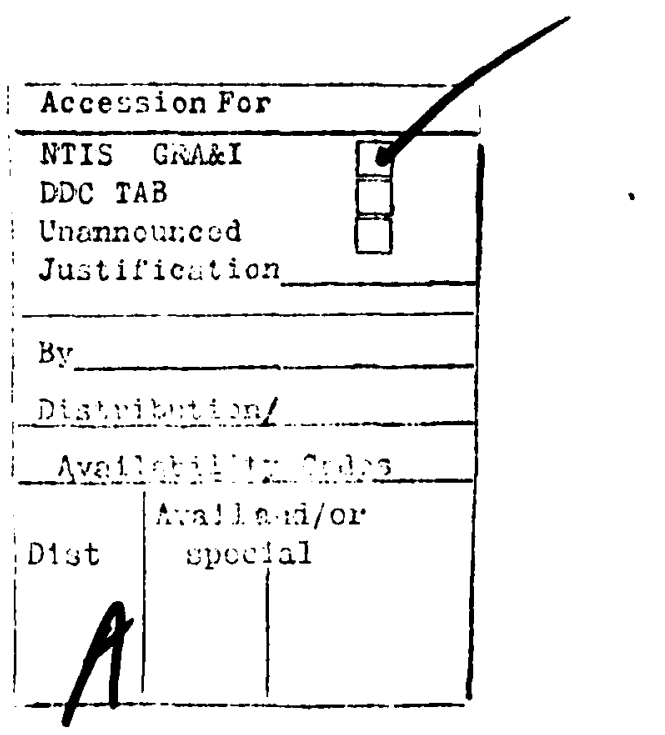




\section{FIGURES}

1. Photon Lifetime Measurement Method . . . . . . . . . 6

2. Tan $(\alpha)$ vs Intracavity Distance ............. 11

3. Tan $(\alpha)$ vs Intracavity Distance .............. 13

4. Three-Mirror Configuration for

Measurement of Photon Lifetime . . . . . . . . . . 15

TABLE

I. Results of Tests on Several Mirrors

of Differing Characteristics .............. 12

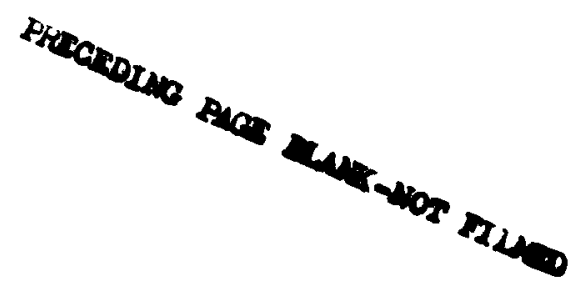




\section{INT RODUCTION}

The precision measurement of optical reflectances or transmittances can be very difficult when either of these quantities approaches unity. ${ }^{1}$ It is, however, in this limit of high reflectance or high transmittance that such measurements can be of great importance, particularly with regard to the design and construction of low-gain laser systems, where the critical parameters are often the difference between a mirror reflectance or window transmittance and unity. In this report, a sensitive, simple laboratory method is presented that can be used to make measurements to a precision of better than $100 \mathrm{ppm}$, thus permitting the testing of mirror reflectivity as high as $99.99 \%$ or the determination of single pass gain or loss coefficients, or window transmission losses, to $10^{-4}$.

The technique described herein can be used to measure the effective photon lifetime in an optical resonator. The effective photon lifetime is defined as the characteristic time $T$ for the photon energy to be dissipated within the optical resonator to $\mathrm{e}^{-1}$ (or 0.34 ) of its original value. The photon is launched into the carefully aligned resonator on the optical axis. The dissipation of photon energy within the resonator is by absorption, scattering, or transmission at the resonator mirror surface coatings or in the medium between. Thus, the technique is a sensitive and useful method for measuring the scattering, absorption, and reflectivity or transmission, or both, of any solid, liquid, or gaseous material that is a part of, or is introduced into, this resonator.

The photon lifetime is determined from the phase shift in the amplitude-modulated photon flux intensity, which has passed through the optical resonator. A fundamental arrangement of the basic components is shown in Fig. 1. A continuous photon source is passed through a piezooptical birefringence modulator or photoelastic modulator (PEM), e.g. , by Morvue, located at position 1, which produces a time-varying, linearly 


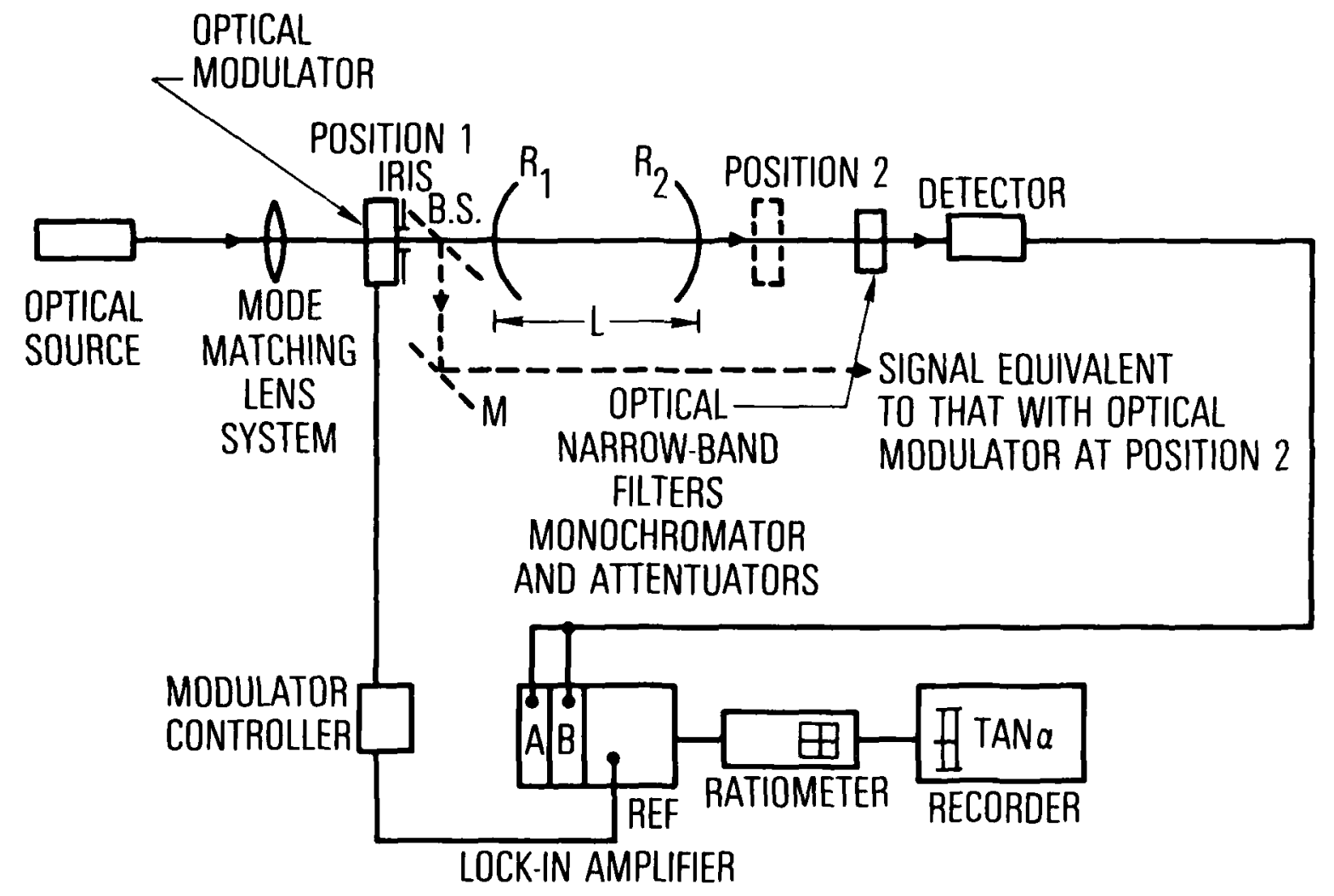

Figure 1. Photon Lifetime Measurement Method 
polarized photon beam with a $\sin ^{2}\left(2 \pi \mathrm{ft} \pm \phi_{0}\right)$ modulation at $f=50 \mathrm{kHz} .^{2}$ This modulated beam is then passed through the optical resonator, which consists of two mirrors. The light beam that emerges from the resonator is also modulated but is shifted in phase by $\alpha$, where the angle $\alpha$ is related to the time the photon spends in the cavity $T$ by the simple expression

$$
\tan (\alpha)=4 \pi f \tau
$$

This lifetime $\tau$ corresponds to some number $n$ of round trips that the photons make within the optical resonator. The time $\tau^{\prime}$ to make one round trip is equal to twice the distance $L$ separating the first and last mirror divided by the speed of light through the medium between mirrors $c$. Therefore

$$
\tau=n \tau^{\prime}=\frac{2 n L}{c}
$$

For large mirror reflectance $R$, this lifetime is related to the overall loss of the optical resonator per pass $1-\mathscr{R}$ by the standard relationship

$$
\mathscr{R}=\exp \left(\frac{-\tau^{\prime}}{\tau}\right)=\exp \left(\frac{-1}{\mathrm{n}}\right)
$$

and for the particular resonator shown in Fig. 1

$$
\mathscr{R}=\mathrm{R}_{1} \mathrm{R}_{2} \leqq 1
$$

The derivation of Eq. (1) is straightforward from the differential equation for flux intensity $I$ at a given wavelength

$$
\frac{d I}{d t}+\frac{I}{\tau}=k_{1} \cos (4 \pi f t)+k_{2}
$$


in which the identity $\sin ^{2}(x)=1 / 2-1 / 2 \cos (2 x)$ is used. The constants $k_{1}$ and $k_{2}$ are of no consequence since they do not enter into the solution for the phase shift. Equation (5) is completely analogous to the equation describing an $\mathrm{RC}$ circuit with an impressed voltage varying as $E_{0} \cos (4 \pi \mathrm{ft})$. For small $n(1<\mathrm{n}<100)$, a more fundamental deviation involving summations rather than integrations is required. This more complicated analysis, given in the Appendix, yields an even simpler expression for $\mathscr{R}$

$$
\mathscr{R}=\frac{\mathrm{n}}{(\mathrm{n}+1)}
$$

It can readily be verified that the expansions of Eqs. (3) and (3a), respectively, differ only in second-order corrections, which are very small for $n \gg 1$. Since Eq. (3a) is simpler, it will be used throughout this discussion. 


\section{EXPERIMENT AND RESULTS}

The experimental apparatus is shown in Fig. 1. The phase shift $\alpha$ can be determined by means of standard lock-in amplifier techniques in several ways. First, the phases resulting from the modulator being placed in position 1 can be measured and then moved to position 2. The difference between these two measured values is the phase shift $\alpha$ resulting from the optical resonator. Second, the modulator can be left in position 1 and a measurement made before and after removing or changing one of the optical elements in the resonator. Again, the difference will yield the change in the lifetime associated with the change in that particular optical element. The third technique requires either two lock-in amplifiers and a ratiometer or a two-channel lock-in amplifier and a ratiomete $r$ or servoloop. (Princeton Applied Research model 5204 is excellent for this application because it has two channels and a ratiometer in one unit.) With the modulator in position 2 , the lock-in(s) is adjusted to the appropriate phase to null out signals on both channels $A$ and $B$. Then, channel B is rotated by $90 \mathrm{deg}$ and the modulator moved to position 1. In this arrangement, the signal in channel $A$ is proportional to the sine of the phase shift $\alpha$, whereas that in channel $B$ is proportional to the cosine of the phase shift $\alpha$. The outputs from these two channels are then fed into the ratiometer, which reads directly

$$
\frac{A}{B}=\frac{\sin (\alpha)}{\cos (\alpha)}=\tan (\alpha)
$$

This technique is convenient for making in situ measurements of optical resonators. The detector gain can be servoed to hold the "in phase" output of the lock-in constant, thereby causing the quadrature channel to read $\tan (\alpha)$ directly. 
The main application of the setup in Fig. 1 is the measurement of mirror reflectances to very high precision. In this configuration, the single-pass reflectance is

$$
\mathscr{R}=\mathrm{R}_{1} \mathrm{R}_{2}
$$

By the measurement of three $\alpha^{\prime}$ 's for the mirrors with reflectances $R_{1}, R_{2}$, and $R_{3}$ in the combinations $R_{1} R_{2}, R_{2} R_{3}$, and $R_{1} R_{3}, R_{1}$ and $R_{2}$ can easily be determined. It is also useful to plot $\tan (\alpha)$ versus $L$ and use the slope of the line to determine the value of $R$. Such plots should be linear and extrapolate to the origin (Fig. 2). We have used this technique to measure the reflectivity of mirrors ranging from $95 \%$ reflecting to $99.98 \%$ reflecting with a precision uncertainty of less than 100 ppm (Fig. 2 and Table I).

Although, in principle, the photon source may be an incoherent, nearly monochromatic, highly collimated beam or a continuous-wave (cw) laser beam of reasonable beam quality, mode matching as well as a spatially coherent source are necessary in order to obtain a linear phasefrequency plot (Fig. 3). The nonlinear phase-frequency response occurs when more than a single mode of the resonant cavity is excited by the laser because the differing longitudinal modes have different photon lifetimes.

In addition, for high-reflectivity mirrors, a laser source is also necessary in order to provide sufficient light on the detector to keep statis tical noise low so that good measurement can be made. There is also a certain amount of noise introduced into the measurement as a result of the thermal agitation of the cavity under test mainly because of length variations caused by acoustic forces; however, with a reasonable time constant, this effect is averaged out in the lock-in amplifier. 


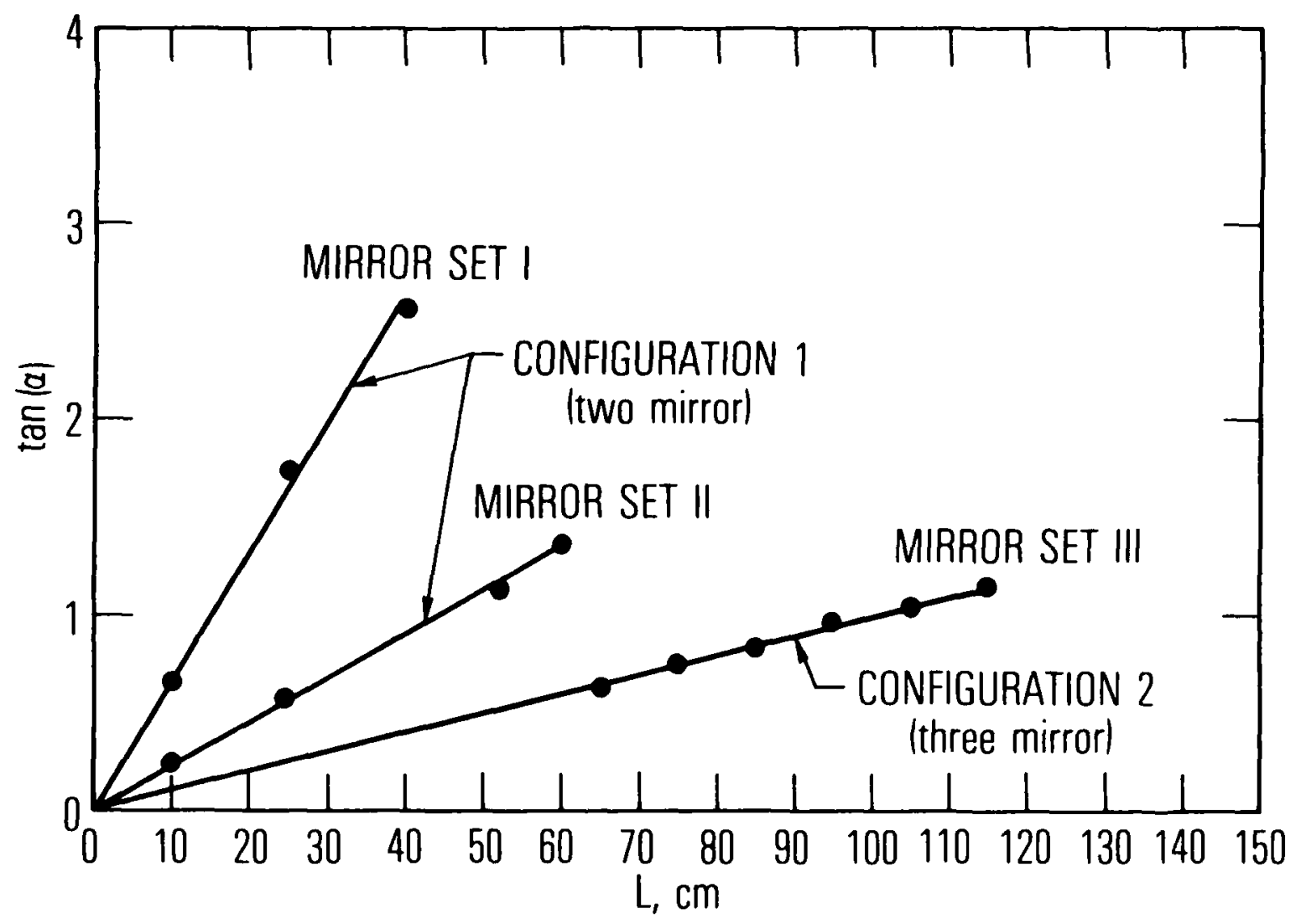

Figure 2. Tan $(\alpha)$ vs Intracavity Distance 
Table 1. Results of Tests on Several Mirrors of Differing Characteristics

\begin{tabular}{ccccccc}
\hline $\begin{array}{c}\text { Mirror } \\
\text { Set }\end{array}$ & & $\mathrm{R}_{1}$ & $\mathrm{R}_{2}$ & $\mathrm{R}_{3}$ & $\begin{array}{c}\text { Mirror Radii } \\
\text { of Curvature }\end{array}$ & $\begin{array}{c}\text { Wavelength, } \\
\AA\end{array}$ \\
\hline I & 0.9994 & 0.9997 & 0.9997 & $2 \mathrm{M}, \infty$ & 8742 \\
II & 0.9981 & 0.9992 & 0.9989 & $6 \mathrm{M}, \infty$ & 6328 \\
III & 0.9957 & 0.9992 & 0.9991 & 0.9987 & $6 \mathrm{M}, 6 \mathrm{M}, \infty$ & 6328 \\
IV & 0.9983 & 0.9992 & 0.9991 & $6 \mathrm{M}, 6 \mathrm{M}$ & 6328 \\
V & 0.9905 & 0.9952 & 0.9952 & $2 \mathrm{M}, 2 \mathrm{M}$ & 5288 \\
VI & 0.9925 & 0.9962 & 0.9962 & $2 \mathrm{M}, \infty$ & 5288 \\
\hline
\end{tabular}




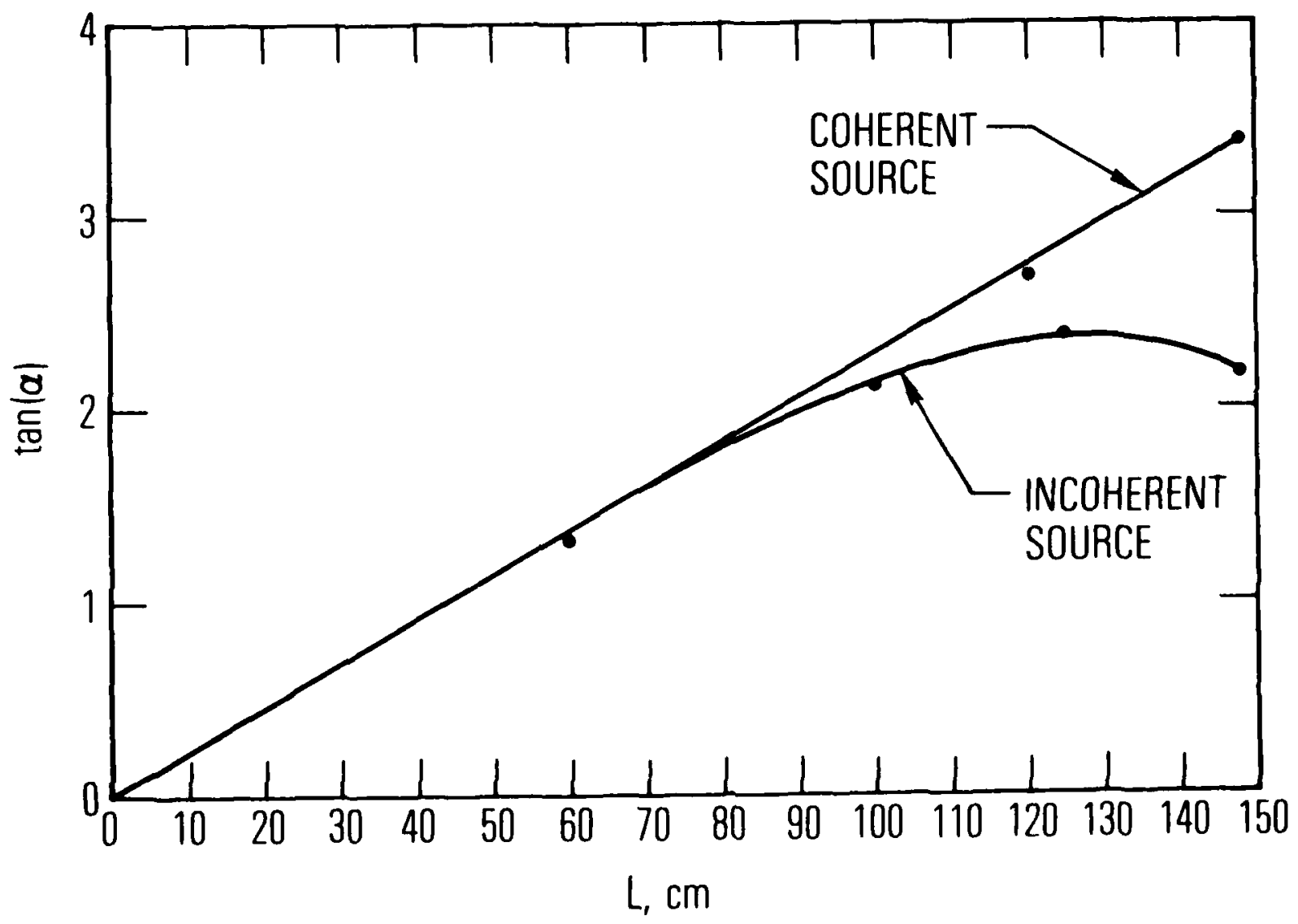

Figure 3. Tan $(\alpha)$ vs Intracavity Distance 
By the removal of $R_{2}$ in Fig. 1 , it is obvious that the transmittance $T$ of $R_{1}$ can be measured by moving $R_{1}$ in and out of the beam and taking the ratio of the two intensities. In general, calibrated attenuation of the detector will be necessary.

A second configuration (Fig. 4), yields an overall single-pass reflectance

$$
\mathscr{R}=\mathrm{R}_{1} \mathrm{R}_{3}^{2} \mathrm{R}_{2}
$$

and permits the measurement of any mirror reflectance $R_{3}$ for a specified polarization when the reflectances of $R_{1}$ and $R_{2}$ have been determined. The apparatus can then be packaged and standardized for measuring reflectances. Furthermore, the measurement can be performed for a range of angles of incidence $\theta / 2$. The lower curve in Fig. 2 was obtained with the use of this three-mirror configuration with $\theta=13 \mathrm{deg}$. The results of tests on several mirrors of differing characteristics a re given in Table $I$. The selfconsistency indicates that the method is accurate within $100 \mathrm{ppm}$. 


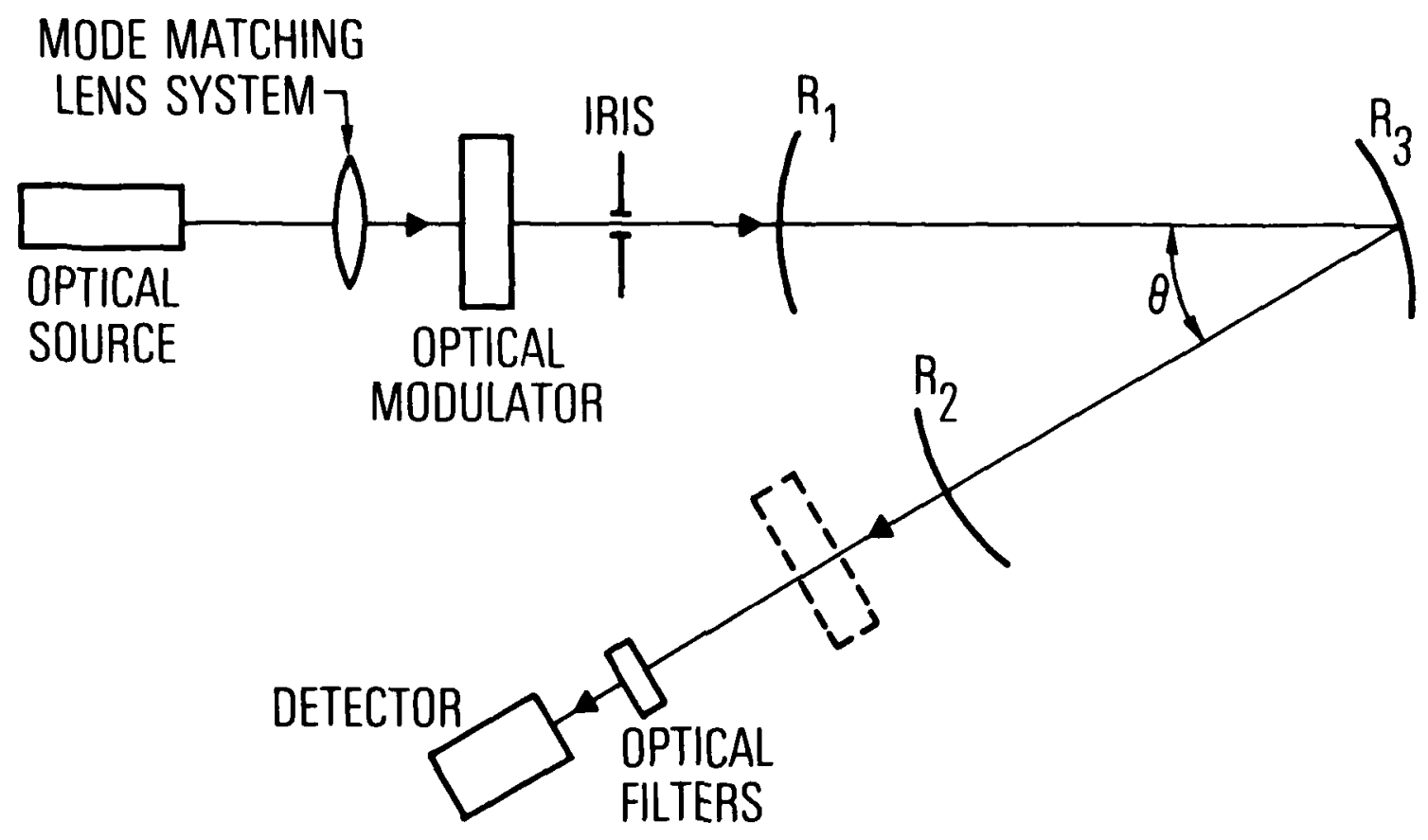

Figure 4. Three-Mirror Configuration for Measurement of Photon Lifetime 


\section{DISCUSSION}

The main advantage of this technique in the determination of reflectances and transmittances is that it can be used for in situ laser cavities as a diagnostic and gain measurement, a very significant factor because the reflectances of ultralow-loss mirrors can easily be altered as a result of handling or transport procedures. Further, once a laser cavity is assembled about a potential gain medium, an in situ cavity finesse experiment can be very valuable with respect to predicting the lase $r$ threshold condition, particularly since subtle losses in the gain medium such as those that might be caused by turbulent scattering can be evaluated with this method. The method is also useful for cavity tuning and alignment as the phase shift will decrease with detuning because of losses caused by beam walk-off and vignetting on apertures.

Many other applications are envisioned, including very sensitive gas phase absorption studies and the study of optically transparent materials under high electric or magnetic fields or both. Similar phase-shift methods have also been routinely used with laser-induced fluorescence to measure excited-state lifetimes in atoms and molecules. 3

The most important application, however, is the use of the method in studying high reflectances and transmittances or very weak absorptances, where the conventional methods fail. Therefore, the phase-shift method can best be applied to an evaluation of low-loss dielectric coatings and Brewster windows for laser applications. The variability of the phase shift with cavity length and modulation frequency, as well as the photon lifetime in general, always makes it practical to arrange an experimental apparatus to permit a significant and easy-to-measure phase shift. The method is quantitatively accurate inasmuch as the only measurements are length, frequency, and phase, and the results are subject to verification by both the linearity of the phase-length plot and self-consistency checks. 


\section{APPENDIX}

The analysis is based on the important assumption that an integration over intensities can be performed rather than the standard summation over amplitudes. The use of intensities is justified by the fact that both the probe laser cavity and the test optical cavities are moving and causing the coherent lengths of the light to be greatly reduced. This variation results in random fluctuation in the output on the microsecond time scale that is the result of constructive and destructive interference. ${ }^{4}$ Since the phase-sensitive measurement is integrated on the second time scale, however, this variation averages out. Consequently, only intensities are of concern.

Let the incoming light to the optical cavity be given by intensity

$$
\underset{\text { in }}{I(t)}=I_{0} \sin \left(4 \pi f t+\phi_{0}\right)
$$

which is equivalent to the $\cos (4 \pi \mathrm{ft})$ used in Eq. (5). The light that is transmitted at time $t$ will be the sum of the intensities from different internal reflectors each reduced by the round-trip reflectivity losses $\mathscr{R}$, i. e. ,

$$
I_{\text {out }}(t)=T I_{0} \sum_{k=1}^{\infty} \cdot R^{k} \sin \left[4 \pi f\left(t-k \tau^{\prime}\right)+\phi_{0}\right]
$$

where $T^{\prime}$ represents the round-trip time, and $T$ is the transmittance after passage through the mirror substrates. It is a reasonably straight-forward exercise to perform the summation of Eq. (A-2) with the following identities $^{5}$

$$
\sum_{k=0}^{\infty} \Re^{k} \sin [(k+1) \theta]=\frac{\sin (\theta)}{1-2 \cdot \Re \cos (\theta)+\mathscr{R}^{2}}
$$


and

$$
\sum_{\mathrm{k}=0}^{\infty} \cdot \mathscr{R}^{\mathrm{k}} \cos [(\mathrm{k}+1) \theta]=\frac{\cos (\theta)-\mathscr{R}}{1-2 \mathscr{R} \cos (\theta)+\mathscr{R}^{2}}
$$

for $\mathscr{R}<1$. By substituting and rearranging, we obtain

$$
I_{\text {out }}=T I_{0} \frac{\sin (\omega t)-\mathscr{R} \sin \left(\omega t+\omega \tau^{\prime}\right)}{1-2 \cdot R \cos \left(\omega T^{\prime}\right)+\cdot R^{2}}
$$

where $\omega \equiv 4 \pi f$ and $\phi_{0}=0$ for convenience. We now use the trigonometric theorem, ${ }^{6}$ which states that

$$
S=a \sin (\omega t+\alpha)+b \sin (\omega t+\beta)=r \sin (\omega t+\phi)
$$

where

$$
r=\left(A^{2}+B^{2}\right)^{1 / 2}, \tan (\phi)=\frac{B}{A}
$$

and

$$
A=a \cos (\alpha)+b \cos (\beta) \text { and } B=a \sin (\alpha)+b \sin (\beta)
$$

The final expression for the transmitted intensity simplifies to

$$
I_{\text {out }}=T_{0} \frac{\sin (\omega t+\phi)}{\left(1-2 \cdot R \cos \left(\omega \tau^{\prime}\right)+. R^{2}\right)^{1 / 2}}
$$


where

$$
\tan (\phi)=\frac{-\mathscr{R} \sin \left(\omega T^{\prime}\right)}{1-\mathscr{R} \cos \left(\omega T^{\top}\right)} \simeq \frac{-\omega T^{\prime} \mathscr{R}}{1-\mathscr{R}} \equiv-n \omega T^{\prime}
$$

Equation (A-8) approximates to the simpler form because, for all cases of interest, $\omega \tau^{\prime} \ll 1$, and, consequently, $\sin \left(\omega \tau^{\prime}\right) \simeq \omega \tau^{\prime}$ and $\cos \left(\omega \tau^{\prime}\right) \simeq 1$. Rearrangement of Eq. $(\mathrm{A}-8)$ results in the simple expression of Eq. (3a). 


\section{REFERENCES}

1. V. Sanders, Appl. Opt. $\underline{16}, 191$ (1977).

2. J. Kemp, J. Opt. Soc. Am. 59, 950 (1969).

3. W. J. Tango and R. N. Zare, J. Chem. Phys. 53, 3094 (1970).

4. M. Born and M. Wolf, Principles of Optics, 4 ed. (Pe rgamon Press, New York, 1970), p. 325.

5. C. R. Wylie, Advances in Engineering Mathematics, 3rd ed., (McGraw-Hill, New York, 1960), p. 697.

6. E. P. Vance, Modern Algebra and Trigonometry (Addison-Wesley 1962), p. 287. 


\section{LABORATORY OPERATIONS}

The Laboratory Operations of The Aerospace Corporation is conducting experimental and theoretical investigations necessary for the evaluation and application of ocientific advances to new military concepte and systems. Vexsatility and flexibility have been developed to a high degree by the laboratory peroonnel in dealing with the many problems encountered in the nation's rapidly developing upace and missile syatems. Expertise in the latest scientific developments is vital to the accomplishment of tasks related to these problems. The laboratories that contribute to this research are:

Aerophyoics Laboratory: Launch and reentry aerodynamics, heat transfer, reentry physics, chemical kinetics, structural mecharics, flight dynamica, atmospheric pollution, and high-power gas lasers.

Chemistry and Physics Laboratory: Atmospheric reactions and atmospheric optics, chemical reactions in polluted atmospheres, chemical reactions of excited species in rocket plumes, chemical thermodynamics. plaeme and laser-induced reactions, laser chemistry, propulsion chemistry, space vacuum and radiation effects on materials, lubrication and urface phenomena, photosensitive materials and sensors. high precision laser ranging. and the application of physics and chemistry to problems of law enforcement and biomedicine.

Electronice Research Laboratory: Electromagnetic theory, devices, and propagation phenomena, including plasma electromagnetics; quantum electronics, lasers, and electro-optics; communication sciences, applied electronics, semiconducting, superconducting. and crystal device physics, optical and acoustical imaging; atmospheric pollution: millimeter wave and far-infrared technology.

Materials Sciences Laboratory: Developinent of new materials; metal matrix composites and new forms of carbon; test and evaluation of graphite and ceramics in reentry; spacecraft materials and electromc components in nuclear weapons environment; application of fracture mechanice to strese corrosion and fatigue-induced fractures in structural metals.

Space Sciences Laboratory: Atmospheric and ionospheric physica, radiation from the atmoophere, density and composition of the atmosphere, aurorae and airglow; magnetospheric physics, cosmic rays, generation and propagation of plasma waves in the magnetosphere; solar physics, studies of solar magnetic fields; space astronomy, $x$-ray astronomy; the effects of nuclear explosions. magnetic storms, and olar activity on the earth's atmosphere, ionosphere. and magnetosphere; the effects of optical, electromagnetic, and particulate radiations in opace on space systems.

THE AEROSPACE CORPORATION

El Segundo, California 